\title{
optica
}

\section{Experimental demonstration of Gaussian protocols for one-sided device-independent quantum key distribution: supplementary material}

\author{
Nathan WalK ${ }^{1,3,4, *}$, Sara Hosseini ${ }^{2}$, JiaO Geng ${ }^{2}$, Oliver Thearle ${ }^{2}$, Jing \\ YAn Haw $^{2}$, SeiJi Armstrong ${ }^{2}$, Syed M. Assad ${ }^{2}$, JiRI JanOUSEK ${ }^{2}$, Timothy C. \\ RALPH $^{1}$, ThOMAS Symul ${ }^{2}$, HoWARd M. Wiseman ${ }^{3}$, ANd Ping KoY LAM ${ }^{2}$ \\ ${ }^{1}$ Centre for Quantum Computation and Communication Technology, School of Mathematics and Physics, University of Queensland, St. Lucia, \\ Queensland 4072, Australia \\ ${ }^{2}$ Centre for Quantum Computation and Communication Technology, Department of Quantum Science, Research School of Physics and \\ Engineering, The Australian National University, Canberra ACT 2601, Australia \\ ${ }^{3}$ Centre for Quantum Computation and Communication Technology, Centre for Quantum Dynamics, Griffith University, Brisbane, Queensland \\ 4111, Australia \\ ${ }^{4}$ Department of Computer Science, University of Oxford, Wolfson Building, Parks Road, Oxford OX1 3QD, United Kingdom \\ *Corresponding author: nathan.walk@cs.ox.ac.uk
}

Published 10 June 2016

\begin{abstract}
This document provides supplementary information to "Experimental demonstration of Gaussian protocols for one-sided device-independent quantum key distribution," http://dx.doi.org/10.1364/ optica.3.000634. For completeness, we first review the necessary entropic uncertainty relations and then derive explicit secret key rate formulae for all the protocols considered. Finally, we present some detailed modelling of the experiment and the effect of potential improvements.
\end{abstract}

(C) 2016 Optical Society of America

http://dx.doi.org/10.1364/optica.3.000634.s001

\section{ENTROPIC UNCERTAINTY RELATIONS}

Entropic relations have received a great deal of attention as a convenient and powerful information theoretic tool for investigating uncertainty in quantum systems. Originally, entropic uncertainty relations were derived assuming one starts without any additional information or at most only classical information describing the system in question, i.e. the density matrix $[1,2]$. In either case, since classical information can be shared perfectly amongst arbitrarily many parties, there is little sense in thinking about these relations as applying from the perspective of one observer or another. Conversely, if observers were to share quantum correlations with the measured system, one expects the uncertainty relations to be strongly observer dependent and potentially exhibit reduced levels of uncertainty.

A generalised relation, allowing for this so-called quantum side information, was derived in [3] although only for finite dimensional Hilbert spaces and observables with a discrete spectrum. Consider a pair of observables $\left\{\hat{x}_{A}, \hat{p}_{A}\right\}$ with a complementarity $c=$ $\max _{p_{A}, x_{A}}\left|\left\langle x_{A} \mid p_{A}\right\rangle\right|^{2}$ where $\left\{\left|x_{A}\right\rangle,\left|p_{A}\right\rangle\right\}$ are the eigenvectors of the observables. These observables are to be measured on a state $A$ which is potentially entangled with another state, $B$, leading to the the following relation for the uncertainty in the pair of observables given access to $B[3]$,

$$
S\left(X_{A} \mid B\right)+S\left(P_{A} \mid B\right) \geq \log \frac{1}{c}+S(A \mid B) .
$$

Here $S(A \mid B)=S(A B)-S(B)$ where $S(X)=-\operatorname{tr}\left(\rho_{X} \log \rho_{X}\right)$ is the conditional von Neumann entropy of the state $\rho_{A B}$ whereas $S\left(X_{A} \mid B\right)$ is the conditional von Neumann entropy of the random variable, $X_{A}$, corresponding to the measurement of the observable $\hat{x}_{A}$ on system $A$ given knowledge of system $B$. This is defined as,

$$
S\left(X_{A} \mid B\right)=H\left(X_{A}\right)+\sum_{x_{A}} p\left(x_{A}\right) S\left(\rho_{B}^{x_{A}}\right)-S(B)
$$

with $H\left(X_{A}\right)=-\sum_{x_{A}} p\left(x_{A}\right) \log p\left(x_{A}\right)$ the Shannon entropy and $\rho_{B}^{x_{A}}$ describing Bob's state conditional on Alice obtaining outcome $x_{A}$. The presence of the conditional entropy $S(A \mid B)$ in Eq. S1, which is negative for entangled states, demonstrates both the observer dependence and effect of entanglement in reducing uncertainty. 
Preempting applications to quantum key distribution (QKD), one can also consider that the state $\rho_{A B}$ could have suffered some decoherence which is purified by an environment, or eavesdropper, such that $\rho_{A B}=\operatorname{tr}_{E}(|A B E\rangle\langle A B E|)$. Using the purity of the overall state (i.e. $S(A B)=S(E))$ one can recast equation (S1) to find [3],

$$
S\left(X_{A} \mid B\right)+S\left(P_{A} \mid E\right) \geq \log \frac{1}{c} .
$$

However, these results are only valid for measurements with a finite number of discrete outcomes made on states living in a finitedimensional Hilbert space. For the purposes of continuous variable (CV) QKD we will require an uncertainty relation valid for infinitedimensional Hilbert spaces and continuous-valued measurements. In particular, we are interested in homodyne measurements of the canonically conjugate quadratures $\hat{x}=\sqrt{\frac{\hbar}{2}}\left(\hat{a}+\hat{a}^{\dagger}\right), \hat{p}=\sqrt{\frac{\hbar}{2}} i\left(\hat{a}^{\dagger}-\hat{a}\right)$ satisfying $[\hat{x}, \hat{p}]=i \hbar$ where $\hat{a}$ and $\hat{a}^{\dagger}$ are bosonic annihilation and creation operators.

Just such a relation has been recently developed, building on an earlier result for discrete and finite measurements on infinite dimensional Hilbert spaces [4]. This was first extended to countably infinite measurements which could then be applied to a discretised version of a homodyne detection [5]. Deriving results for continuous spectra, by taking infinite precision limits of these coarse-grained POVM's, had previously been extensively studied for the Shannon entropies, and an analogous procedure for the quantum conditional von Neumann entropy was utilised by Ferenczi [6] and Berta et al. [5] although the former proof is incomplete. An alternative derivation was also provided by Frank and Lieb [7]. The final result is the following relation for homodyne detection upon infinite dimensional Hilbert spaces [5-7],

$$
S\left(X_{A} \mid B\right)+S\left(P_{A} \mid E\right) \geq \log 2 \pi \hbar
$$

\section{SECRET KEY RATES FOR GAUSSIAN PROTOCOLS}

We will now derive bounds upon the secret key rate for all members of the Gaussian family of CVQKD protocols. In the P\&M setting, one could consider Alice sending either squeezed [8,9] or coherent states [10] and Bob measuring with either homodyne or heterodyne $[11,12]$ detection. Each permutation can in turn be used to have Bob try and guess Alice's encoding, called direct reconciliation [13] (DR), or Alice trying to guess Bob's measurement [10], called reverse reconciliation (RR). One can also consider entanglement based (EB) schemes, in which Alice distributes one arm of a two-mode squeezed vacuum to Bob [14]. In fact, with appropriate rescaling of Alice's data, the EB and $\mathrm{P} \& \mathrm{M}$ schemes can be seen as equivalent since having Alice heterodyne detect one arm of a two-mode squeezed vacuum corresponds to preparing a coherent state, while a homodyne detection corresponds to squeezed state preparation [15]. In the following, we conduct our analysis in the EB picture, and the variances appearing are those that would be directly measured in an EB implementation. We will calculate the key rate encoded in the $\hat{x}$ basis on a particular run. Overall, the total key rate will be the average of the quantities derived here and the analogous expression for encoding in the $\hat{p}$ basis (the exception is the heterodyne-heterodyne protocol where both bases are always simultaneously utilised). Although we calculate all the key rates for completeness, for the protocols involving heterodyne detection only one reconciliation direction will allow for a $1 \mathrm{sDI}$ protocol as per the main text.

\section{A. Homodyne-Homodyne (Squeezed states and Homodyne Detection)}

First we turn to the protocols where both Alice and Bob homodyne detect. Here Alice and Bob randomly choose whether they measure the $\hat{x}$ or $\hat{p}$ basis and only keep those times where they agree. To obtain effective EB data from a P\&M scheme Alice will rescale her modulation signal by dividing $\alpha_{x(p)}=+(-) \sqrt{1-1 / V_{x(p)}^{2}}$ where $V_{x(p)}=V_{S_{x(p)}}+1$ and $V_{S_{x(p)}}$ is the modulation variance of the signal encoded in the respective quadratures. We work in the asymptotic regime $n \rightarrow \infty$ where the optimal attacks are known to be the collective attacks. Considering the case where Bob makes an $\hat{x}$ measurement, we can write the secret key rate for the DR protocol as $[16,17]$,

$$
K^{\triangleright} \geq I\left(X_{A}: X_{B}\right)-\chi\left(X_{A}: E\right)
$$

where

$$
\begin{aligned}
I\left(X_{A}: X_{B}\right) & =H\left(X_{B}\right)-H\left(X_{B} \mid X_{A}\right) \\
& =H\left(X_{A}\right)-H\left(X_{A} \mid X_{B}\right)
\end{aligned}
$$

denotes the classical mutual information between Alice and Bob, with $H(X)=-\int d x p(x) \log p(x)$ being the continuous Shannon entropy of the measurement strings and

$$
\chi\left(X_{A}: E\right)=S(E)-\int d x_{A} p\left(x_{A}\right) S\left(E \mid x_{A}\right)
$$

is the Holevo bound.

Substituting equations (S6) and (S7) into (S5) and comparing with the continuous conditional von Neumann entropy

$$
S\left(X_{A} \mid B\right)=H\left(X_{A}\right)+\int d x_{A} p\left(x_{A}\right) S\left(\rho_{B}^{x_{A}}\right)-S(B)
$$

we have,

$$
\begin{aligned}
K^{\triangleright} & \geq H\left(X_{A}\right)+\int d x_{A} p\left(x_{A}\right) S\left(\rho_{E}^{x_{A}}\right)-S(E)-H\left(X_{A} \mid X_{B}\right) \\
& =S\left(X_{A} \mid E\right)-H\left(X_{A} \mid X_{B}\right)
\end{aligned}
$$

which is what one would expect from the Devetak-Winter relations [18]. Using the entropic uncertainty relation we can bound the eavesdropper's information on the relevant observable. Substituting from (S4) and recalling that $S\left(P_{A} \mid B\right) \leq S\left(P_{A} \mid P_{B}\right)=H\left(P_{B} \mid P_{A}\right)$, we can write,

$$
\begin{aligned}
K^{\triangleright} & \geq \log 4 \pi-H\left(X_{A} \mid X_{B}\right)-S\left(P_{A} \mid B\right) \\
& =\log 4 \pi-H\left(X_{A} \mid X_{B}\right)-H\left(P_{A} \mid P_{B}\right) .
\end{aligned}
$$

As discussed in the main text, Alice and Bob can bound their secret key rate for this protocol by measuring Bob's conditional variance. Substituting the Shannon entropy for a Gaussian distribution $H_{G}\left(X_{B} \mid X_{A}\right)=\log \sqrt{2 \pi e V_{X_{B} \mid X_{A}}}$, where $V_{X_{B} \mid X_{A}}=V_{X_{B}}-$ $\left\langle X_{A} X_{B}\right\rangle^{2} / V_{X_{A}}$, we arrive at a final expression for the key rate of

$$
\begin{aligned}
K^{\triangleright} & \geq \log 4 \pi-\log 2 e \pi \sqrt{V_{X_{A} \mid X_{B}} V_{P_{A} \mid P_{B}}} \\
& =\log \left(\frac{2}{e \sqrt{V_{X_{A} \mid X_{B}} V_{P_{A} \mid P_{B}}}}\right)
\end{aligned}
$$

The RR expression is obtained by simply permuting the labels of Alice and Bob. That this result is pessimistic is implied by consideration of the predictions for a perfect channel. Under the security proofs presented in [16-18] if Alice and Bob share a pure EPR state (real or effective) with variance $V=\cosh (2 s)$ then the key rate given in (S9) is always positive provided the squeezing parameter is nonzero $(s>0)$. On the contrary, the result in (S11) is only positive for $V_{X_{A} \mid X_{B}} V_{P_{A} \mid P_{B}}=\mathcal{E}_{\triangleleft} \leq\left(\frac{2}{e}\right)^{2} \approx 0.55$ which implies a squeezing parameter of $s \geq .15$ or about $-1.3 \mathrm{~dB}$ of squeezing. This result 
was also calculated in [6], however as mentioned before the proof relied on the assumption of the applicability of the entropic uncertainty relation to infinite dimensional Hilbert spaces. Furthermore, Ferenczi argued that since for coherent states the directly measured conditional variances are always greater than 1 , the above procedure would never predict a positive key rate for coherent state protocols [6]. However, this conclusion comes from a mistaken application of the key rate formulae as we now demonstrate.

\section{B. Heterodyne-Homodyne (Coherent States and Homodyne Detection)}

Consider a DR coherent state protocol, which in the EB picture involves Alice making a heterodyne detection upon her arm of an EPR pair. Thus she first mixes her mode with vacuum resulting in two modes $A_{1}$ and $A_{2}$ upon which she measured $\hat{x}$ and $\hat{p}$ respectively. To obtain effective EB data from a $\mathrm{P} \& \mathrm{M}$ scheme Alice will rescale her modulation signal by dividing $\alpha_{x(p)}=+(-) \sqrt{2\left(V_{x(p)}-1\right) /\left(V_{x(p)}+1\right)}$ where $V_{x(p)}=V_{S_{x(p)}}+1$ and $V_{S_{x(p)}}$ is the modulation variance of the signal encoded in the respective quadratures. Bob then makes a homodyne detection, randomly switching between the quadratures. The DR key rate is then bounded by,

$$
K^{\triangleright} \geq S\left(X_{A_{1}} \mid E\right)-H\left(X_{A_{1}} \mid X_{B}\right)
$$

After Alice's projective measurement upon $A_{1}$ the state $\rho_{A_{1} B E}$ is pure and we can again apply the entropic uncertainty relation to write,

$$
\begin{aligned}
K^{\triangleright} & \geq \log 4 \pi-S\left(P_{A_{1}} \mid B\right)-H\left(X_{A_{1}} \mid x_{B}\right) \\
& \geq \log 4 \pi-H\left(P_{A_{1}} \mid P_{B}\right)-H\left(X_{A_{1}} \mid X_{B}\right)
\end{aligned}
$$

Now this formula might pose a problem, in that we do not measure $\hat{p}$ upon mode $A_{1}$. Nevertheless, this can be circumvented if we trust the devices, specifically the beamsplitter in Alice's station. As noted earlier, we may safely bound the Shannon entropy by assuming that the statistics are Gaussian and therefore only dependent upon the conditional variance i.e. $H\left(P_{A_{1}} \mid P_{B}\right)=\log \sqrt{2 \pi e V_{P_{A_{1}} \mid P_{B}}}$. Then, if we trust Alice's beamsplitter we know that $V_{P_{A_{1}} \mid P_{B}}=V_{P_{A_{2}} \mid P_{B}}$ and thus $H\left(P_{A_{1}} \mid P_{B}\right)=H\left(P_{A_{2}} \mid P_{B}\right)$ which is directly measured. We therefore have,

$$
\begin{aligned}
K^{\triangleright} & \geq \log 4 \pi-\log \sqrt{2 \pi e V_{P_{A_{2}} \mid P_{B}}}-\log \sqrt{2 \pi e V_{X_{A_{1}} \mid X_{B}}} \\
& =\log \frac{2}{e \sqrt{V_{X_{A_{1}} \mid X_{B} V_{P_{A_{2}} \mid P_{B}}}}}
\end{aligned}
$$

Note that for positive key we now require the condition $V_{X_{A_{1}} \mid X_{B}} V_{P_{A_{2}} \mid P_{B}} \leq 0.55$. To illustrate how correlated a state we now require respect to the purely homodyne protocols we rewrite this as a condition on the homodyne variances. Assuming quadrature symmetry $\left(V_{X_{A} \mid X_{B}}=V_{P_{A} \mid P_{B}}\right)$ and using the relationship $2 V_{X_{A_{1}} \mid X_{B}}=$ $V_{X_{A} \mid X_{B}}+1$ (similarly for $\hat{p}$ quadrature), we find the requirement on the heterodyne-homodyne conditional variance is equivalent to the homodyne-homodyne condition $V_{X_{A} \mid X_{B}} V_{P_{A} \mid P_{B}} \leq 0.22$.

For RR the key rate is bounded by,

$$
\begin{aligned}
K^{\triangleleft} & \geq S\left(X_{B} \mid E\right)-H\left(X_{B} \mid X_{A_{1}}\right) \\
& \geq \log 4 \pi-S\left(P_{B} \mid A\right)-H\left(X_{B} \mid X_{A_{1}}\right) \\
& \geq \log 4 \pi-H\left(P_{B} \mid P_{A}\right)-H\left(X_{B} \mid X_{A_{1}}\right) \\
& \geq \log \frac{2}{e \sqrt{V_{P_{B} \mid P_{A}} V_{X_{B} \mid X_{A_{1}}}}}
\end{aligned}
$$

Once again, the quantity $V_{P_{B} \mid P_{A}}$ is not measured directly, but provided we trust Alice's beamsplitter we have $V_{P_{B} \mid P_{A}}=V_{P_{B}}-$ $2\left\langle P_{A_{2}} P_{B}\right\rangle^{2} /\left(2 V_{P_{A_{2}}}-1\right)$ allowing us to evaluate the bound. However, since we must trust Bob's devices in order to apply the entropic uncertainty relation we explicitly may not trust Alice's devices. Therefore, this RR protocol could never be $1 \mathrm{sDI}$.

\section{Homodyne-Heterodyne (Squeezed States and Heterodyne Detection)}

These protocols are essentially mirror images of the coherent state homodyne schemes since, in the EB picture, we now have Alice making homodyne measurements and Bob making heterodyne measurements. We now have Alice swapping between $\hat{x}$ and $\hat{p}$ measurements while Bob splits up his mode measuring $\hat{x}$ upon $B_{1}$ and $\hat{p}$ upon $B_{2}$. As per the previous squeezed state case, to obtain effective EB data from a P\&M scheme Alice will rescale her modulation signal by dividing $\alpha_{x(p)}=+(-) \sqrt{1-1 / V_{x(p)}^{2}}$. The DR key rate is bounded by,

$$
\begin{aligned}
& K^{\triangleright} \geq S\left(x_{A} \mid E\right)-H\left(X_{A} \mid X_{B_{1}}\right) \\
& \geq \log 4 \pi-S\left(P_{A} \mid B\right)-H\left(X_{A} \mid X_{B_{1}}\right) \\
& \geq \log 4 \pi-H\left(P_{A} \mid P_{B}\right)-H\left(X_{A} \mid X_{B_{1}}\right) \\
& \geq \log \frac{2}{e \sqrt{V_{P_{A} \mid P_{B}} V_{X_{A} \mid X_{B_{1}}}}}
\end{aligned}
$$

where we will have to trust the beamsplitter in Bob's station to obtain $V_{P_{A} \mid P_{B}}=V_{P_{A}}-2\left\langle P_{B_{2}} P_{A}\right\rangle^{2} /\left(2 V_{P_{B_{2}}}-1\right)$ from the directly measured conditional variance.

The RR key rate is bounded by,

$$
\begin{aligned}
K^{\triangleleft} & \geq S\left(X_{B_{1}} \mid E\right)-H\left(X_{B_{1}} \mid X_{A}\right) \\
& \geq \log 4 \pi-S\left(P_{B_{1}} \mid A\right)-H\left(X_{B_{1}} \mid X_{A}\right) \\
& \geq \log 4 \pi-H\left(P_{B_{1}} \mid P_{A}\right)-H\left(X_{B_{1}} \mid X_{A}\right) \\
& \geq \log \frac{2}{e \sqrt{V_{P_{B_{2}} \mid P_{A} V_{X_{B_{1}} \mid X_{A}}}}}
\end{aligned}
$$

where we have again used the known action of Bob's beamsplitter to write $V_{P_{B_{1}} \mid P_{A}}=V_{P_{B_{2}} \mid P_{A}}$. Similarly to previous section, only the RR protocol is $1 \mathrm{sDI}$.

\section{Heterodyne-Heterodyne (Coherent States and Heterodyne Detection)}

The final protocols involve Bob making a heterodyne measurement upon coherent states, or alternatively both parties making heterodyne measurements upon a two-mode squeezed vacuum. We include this for completeness, however none of these protocols could be 1sDI because devices on both sides must always be trusted. Thus there are now four modes involved $A_{1}$ and $B_{1}$ upon which $\hat{x}$ is measured and $A_{2}$ and $B_{2}$ upon which $\hat{p}$ is measured. We can consider the $\hat{x}$ and $\hat{p}$ channels separately. Note that this is actually an underestimation of the key rate as it essentially allowing Eve to devote all her resources to estimating either the $\hat{x}$ or $\hat{p}$ measurements separately, whereas in reality she must in fact estimate both simultaneously. As per the previous coherent state case, to obtain effective EB data from a P\&M scheme Alice will rescale her modulation signal by dividing $\alpha_{x(p)}=$ $+(-) \sqrt{2\left(V_{x(p)}-1\right) /\left(V_{x(p)}+1\right)}$. The DR key rate for $\hat{x}$ is bounded 
by,

$$
\begin{aligned}
K^{\triangleright} & \geq S\left(X_{A_{1}} \mid E\right)-H\left(X_{A_{1}} \mid X_{B_{1}}\right) \\
& \geq \log 4 \pi-S\left(P_{A_{1}} \mid B\right)-H\left(X_{A_{1}} \mid X_{B_{1}}\right) \\
& \geq \log 4 \pi-H\left(P_{A_{1}} \mid P_{B}\right)-H\left(X_{A_{1}} \mid X_{B_{1}}\right) \\
& \geq \log \frac{2}{e \sqrt{V_{P_{A_{1}} \mid P_{B}} V_{X_{A_{1}} \mid X_{B_{1}}}}}
\end{aligned}
$$

Provided we trust the beamsplitter in Bob's station we can have $V_{P_{A_{1}} \mid P_{B}}=V_{P_{A_{2}} \mid P_{B}}=V_{P_{A_{2}}}-2\left\langle P_{A_{2}} P_{B_{2}}\right\rangle^{2} /\left(2 V_{P_{B_{2}}}-1\right)$.

The RR key rate is bounded by,

$$
\begin{aligned}
K^{\triangleleft} & \geq S\left(X_{B_{1}} \mid E\right)-H\left(X_{B_{1}} \mid X_{A_{1}}\right) \\
& \geq \log 4 \pi-S\left(P_{B_{1}} \mid A\right)-H\left(X_{B_{1}} \mid X_{A_{1}}\right) \\
& \geq \log 4 \pi-H\left(P_{B_{1}} \mid P_{A}\right)-H\left(X_{B_{1}} \mid X_{A_{1}}\right) \\
& \geq \log \frac{2}{e \sqrt{V_{P_{B_{1}} \mid P_{A}} V_{X_{B_{1}} \mid X_{A_{1}}}}}
\end{aligned}
$$

By trusting the beamsplitter in Alice and Bob's station we get $V_{P_{B_{1}} \mid P_{A}}=V_{P_{B_{2}} \mid P_{A}}=V_{P_{B_{2}}}-2\left\langle P_{B_{2}} P_{A_{2}}\right\rangle^{2} /\left(2 V_{P_{A_{2}}}-1\right)$.

\section{SECURITY PROOF WITH IMPERFECT RECONCILIA- TION EFFICIENCY}

In the main text we derived secret key rates assuming that Alice and Bob achieve the Shannon capacity for their Gaussian encoding. Thus the key rate is bounded by,

$$
K^{\triangleright} \geq I\left(X_{A}: X_{B}\right)-\chi\left(X_{A}: E\right)
$$

Here we now use $X_{A}$ to denote that fact that depending upon the particular protocol we could be referring to quadrature measurements with or without a shot noise penalty and also that these quantities must be averaged over the $\hat{x}$ and $\hat{p}$ quadratures in the case that the two are not perfectly symmetric. In reality we won't be able to perfectly achieve this capacity and the key rate will instead be bounded by,

$$
K^{\triangleright} \geq \beta I\left(X_{A}: X_{B}\right)-\chi\left(X_{A}: E\right)
$$

where $\beta<1$ is the reconciliation efficiency. In this case, instead of using the entropic uncertainty relation to lower bound the secret key rate, we will use it to upper bound Eve's information and then independently measure $\beta I\left(X_{A}: X_{B}\right)$ to obtain the actual key rate. Eve's information is upper bounded by the Holevo quantity thus,

$$
\chi\left(X_{A}: E\right) \leq S(E)-\int d X_{A} p\left(X_{A}\right) S\left(\rho_{E}^{X_{A}}\right)
$$

The conditional von Neuman entropy of the observable $X_{A}$ is given by

$$
S\left(X_{A} \mid E\right)=H\left(X_{A}\right)+\int d X_{A} p\left(X_{A}\right) S\left(\rho_{E}^{X_{A}}\right)-S(E)
$$

Thus we can rewrite Eve's information as,

$$
\chi\left(X_{A}: E\right) \leq H\left(X_{A}\right)-S\left(X_{A} \mid E\right)
$$

We now make use of our CV entropic uncertainty relation,

$$
S\left(X_{A} \mid E\right)+S\left(P_{A} \mid B\right) \geq \log 4 \pi
$$

to obtain,

$$
\chi\left(X_{A}: E\right) \leq H\left(X_{A}\right)+S\left(P_{A} \mid B\right)-\log 4 \pi
$$

Using the fact that $S\left(P_{A} \mid B\right) \leq S\left(P_{A} \mid P_{B}\right)=H\left(P_{A} \mid P_{B}\right)$ and that the Shannon entropy is maximised by a Gaussian distribution for a fixed variance such that $H\left(X_{A}\right) \leq \log \sqrt{2 \pi e V_{X_{A}}}$ we arrive finally at,

$$
\chi\left(X_{A}: E\right) \leq \log 2 \pi e \sqrt{V_{X_{A}} V_{P_{A} \mid P_{B}}}-\log 4 \pi
$$

Thus the secret key rate for an arbitrary $\beta$ is ,

$$
K^{\triangleright} \geq \beta \log \sqrt{\frac{V_{X_{A}}}{V_{X_{A} \mid X_{B}}}}+\log \frac{2}{e \sqrt{V_{X_{A}} V_{P_{A} \mid P_{B}}}}
$$

The RR key rate is given by interchanging Alice and Bob to obtain,

$$
K^{\triangleleft} \geq \beta \log \sqrt{\frac{V_{X_{B}}}{V_{X_{B} \mid X_{A}}}}+\log \frac{2}{e \sqrt{V_{X_{B}} V_{P_{B} \mid P_{A}}}}
$$

Finally, the key rate for the RR protocol where Bob heterodynes is given by

$$
K^{\triangleleft} \geq \beta \log \sqrt{\frac{V_{X_{B_{1}}}}{V_{X_{B_{1}} \mid X_{A}}}}+\log \frac{2}{e \sqrt{V_{X_{B_{1}}} V_{P_{B_{2}} \mid P_{A}}}}
$$

and the DR protocol where Alice heterodynes (or alternatively prepares coherent states) is given by

$$
K^{\triangleright} \geq \beta \log \sqrt{\frac{V_{X_{A_{1}}}}{V_{X_{A_{1}} \mid X_{B}}}}+\log \frac{2}{e \sqrt{V_{X_{A_{1}}} V_{P_{A_{2}} \mid P_{B}}}}
$$

\section{EXPERIMENTAL DETAILS AND MODELING OF EB SCHEME WITH HOMODYNE-HOMODYNE DETEC- TION}

In this section, we discuss the experimental details, the imperfections and the modelling of the experiment with EB source and homodynehomodyne measurements. A $1064 \mathrm{~nm} \mathrm{Nd:YAG} \mathrm{laser} \mathrm{source} \mathrm{which} \mathrm{was}$ frequency doubled to $532 \mathrm{~nm}$ was used in the experiment. Both light fields were passed through mode cleaning cavities for spatial filtering and also to provide quantum noise limited coherent beams. Two similar degenerate bow-tie optical parametric amplifiers were used to produce two amplitude squeezed states. The $1064 \mathrm{~nm}$ field was used as the seed field for the OPAs and also as the local oscillator for homodyne detections. The $532 \mathrm{~nm}$ field was used to pump the OPAs. Here, we used PPKTP crystal to produce nonlinear effects. The estimated values of squeezing and anti-squeezing generated from both OPAs were -6 $\mathrm{dB}$ and $10.7 \mathrm{~dB}$ at $3 \mathrm{MHz}$. A simple model was used to infer the pure squeezing produced inside the cavity and also the effective loss of the system. According to this model, the OPAs were assumed to produce pure squeezed states and the effective loss, the combination OPA's propagation and detectors' losses, was modelled with a beam splitter after the squeezer. Using this model and the measured values of the squeezing and anti-squeezing, we predicted that each OPA produced $11.5 \mathrm{~dB}$ of pure squeezing $(-11.5 \mathrm{~dB}$ of squeezing and $11.5 \mathrm{~dB}$ of anti-squeezing), and the effective loss of this system was calculated to be around $20 \%$ with $14 \%$ loss due to each squeezer's cavity and also the propagation of the optical beams through the optical components and nearly $6 \%$ loss due to each detection station.

Four identical photodiodes were used in the detection stations. The detection efficiency of Alice and Bob's stations were estimated to be $94 \%$ and $92 \%$ respectively, with fringe visibility of $99 \%$ and the photodiodes' quantum efficiency of around $96 \%$ for all the detectors. We estimated 2\% extra loss on Bob's side due to the loss introduced 
(a)

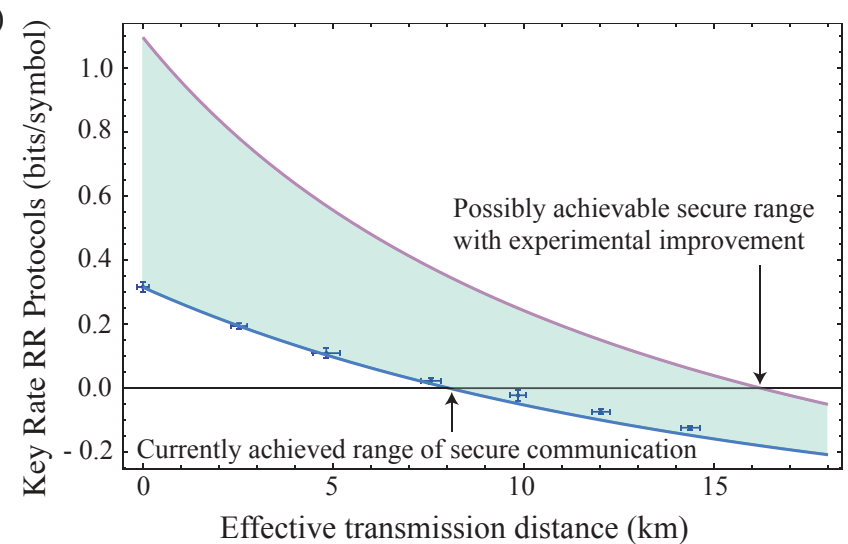

(b)

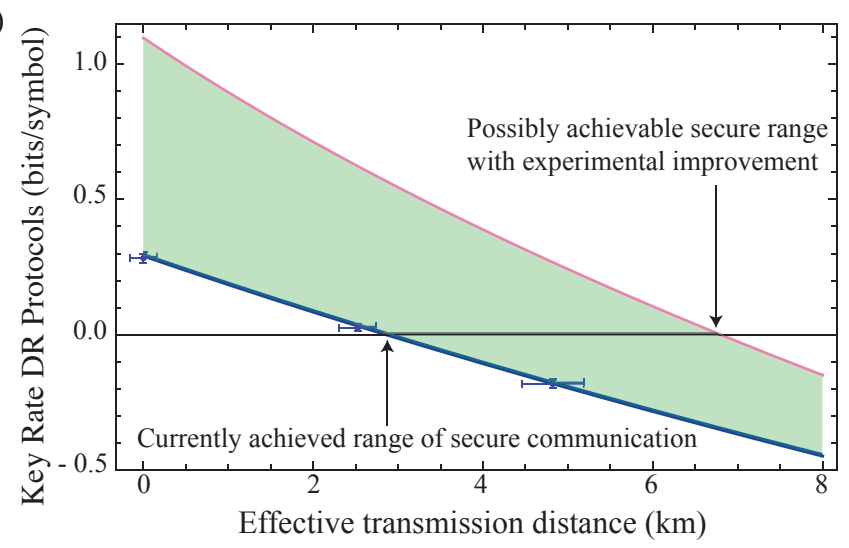

Fig. S1. Predicted improvement of secure transmission distance through optical fibre for the EB protocols with an improved experimental setup (red curve) for the (a) RR protocol and (b) DR protocol. The model for the current system (blue curve) is plotted along with experimental data (blue points) for comparison. The two OPAs in our system each produced $-6 \mathrm{~dB}$ of squeezing and $10.7 \mathrm{~dB}$ of anti-squeezing and suffered from $13 \%$ combined loss due to the squeezers' cavities and the propagation through the optical components. A further loss of $6 \%$ and $8 \%$ was due to the inefficiency of Alice and Bob's homodyne detections respectively. The value of the unknown rotation, $\theta$, was estimated to be $3 \pi / 180$. The improved system consists of two squeezers each producing $-10 \mathrm{~dB}$ of measurable squeezing and $16 \mathrm{~dB}$ of anti-squeezing, $5 \%$ loss due to the cavities and propagation of the optical beams through the optical components with 4\% and 5\% loss for Alice and Bob's stations respectively and a rotation of $\pi / 120$. Reconciliation efficiency is chosen to be 0.95 for both cases. These theoretical lines are produced using the model described in the section 4 and equations (S28) and (S29).

by the half wave plate and polarizing beamsplitter that were used to simulate the lossy channel. Each pair of detectors were balanced electronically, providing $30 \mathrm{~dB}$ of common mode rejection. Each detector had at least $16 \mathrm{~dB}$ of dark noise clearance.

For each separate homodyne detection $5 \times 10^{7}$ data points are sampled at $14 \times 10^{6}$ samples per second. In order to provide sufficient statistics for each data point, this process is repeated ten times. These data were then digitally filtered to $2.5-3.5 \mathrm{MHz}$ and then resampled. After this process, the number of data points was reduced to $4 \times 10^{6}$ which is sufficient to extract the key rates.

Other experimental imperfections, such as the the imperfect locking points and the unbalanced beamsplitter ratios, were also captured in the model. Since all the states and operators were assumed to be Gaussian in this experiment, the states can be easily described by their mean values and covariance matrices (CM's). The effect of Gaussian operations on Gaussian states can be compactly calculated via symplectic transformations [19]. Under an arbitrary symplectic operation, $S$, an input $\mathrm{CM}, \gamma_{\text {in }}$ transforms via

$$
\gamma_{\text {out }}=S \gamma_{\text {in }} S^{T}
$$

The CM of a two mode squeezed vacuum with squeezing in quadrature in modes $i$ and $j$ is given by applying the following symplectic operator,

$$
S Q_{i, j}\left(s_{1}, s_{2}\right)=\left(\begin{array}{cccc}
e^{s_{1}} & 0 & 0 & 0 \\
0 & e^{-s_{1}} & 0 & 0 \\
0 & 0 & e^{-s_{2}} & 0 \\
0 & 0 & 0 & e^{s_{2}}
\end{array}\right)
$$

where $s_{1}$ and $s_{2}$ are squeezing parameters applied on the $i^{\text {th }}$ and $j^{\text {th }}$ second mode respectively. Implicit in this notation is the fact that when applied to a multi-mode CM one should appropriately pad out the above matrix such that the identity is applied to all modes other than $i$ and $j$.

The loss of each squeezer is modelled by introducing a vacuum mode, and then applying a beamsplitter of transmittance $\eta_{A(B)}$ on each squeezed mode and a vacuum mode to mix them together. The beamsplitter transformation between the modes $i$ and $j$ is:

$$
B S_{i, j}(\eta)=\left(\begin{array}{cccc}
\sqrt{\eta} & 0 & -\sqrt{1-\eta} & 0 \\
0 & \sqrt{\eta} & 0 & -\sqrt{1-\eta} \\
\sqrt{1-\eta} & 0 & \sqrt{\eta} & 0 \\
0 & \sqrt{1-\eta} & 0 & \sqrt{\eta}
\end{array}\right)
$$

In order to create an EPR state two squeezed states are locked in quadrature and mixed on a 50:50 beamsplitter. To model the imperfect locking point a phase shift $\theta$ is applied to one mode before they mix on a beamsplitter. The applied operator is as follows:

$$
R T_{i}(\theta)=\left(\begin{array}{cc}
\cos \theta & -\sin \theta \\
\sin \theta & \cos \theta
\end{array}\right)
$$

To model the loss of the transmission channel, a vacuum state was introduced and mixed with the second mode on a beamsplitter with transmittance $T$. The loss of each homodyne station was modelled by a beamsplitter of transmittance $\eta_{D_{A(B)}}$, equal to the efficiency of the homodyne station, with the other mode being in a thermal state of variance $V_{\Delta_{A(B)}}=1+\Delta_{A(B)} /\left(1-\eta_{D_{A(B)}}\right)$ to model the detector dark noise of magnitude $\Delta_{A(B)}$. Thus the final CM is given by (S32) with,

$$
\begin{aligned}
S= & B S_{2,7}\left(\eta_{D_{B}}\right) B S_{2,6}(T) B S_{1,5}\left(\eta_{D_{A}}\right) B S_{1,2}(1 / 2) R T_{2}(\theta) \\
& B S_{2,4}\left(\eta_{B}\right) B S_{1,3}\left(\eta_{A}\right) S Q_{1,2}\left(s_{1}, s_{2}\right)
\end{aligned}
$$

with

$$
\gamma_{\text {in }}=\operatorname{diag}\left(1,1,1,1,1,1,1,1, V_{\Delta_{A}}, V_{\Delta_{A}}, 1,1, V_{\Delta_{B}}, V_{\Delta_{B}}\right)
$$

a 14x14 diagonal matrix. To determine the value of the applied loss, $T$, from the measured correlations it is sufficient to consider the ratio of the correlation between Alice and Bob at particular loss setting with 
the case where the channel is set to full transmission. Using equations (S28) and (S29), key rates were calculated from both the simulated covariance matrix and experimentally measured conditional variances. We plotted them as a function of the effective transmission distance, showing excellent agreement with the experimental results as shown in Fig S1.

The model was also used to estimate the performance of a more efficient system with two squeezers each producing $-10 \mathrm{~dB}$ of squeezing and $16 \mathrm{~dB}$ of anti-squeezing and detection efficiency for Alice and Bob's stations of $96 \%$ and $95 \%$ respectively. Using these parameters, our model shows that the range of the secure communication would extend from $7.5 \mathrm{~km}$ to $15 \mathrm{~km}$ for the RR protocol and from $2.5 \mathrm{~km}$ to more than $6 \mathrm{~km}$ for DR protocol. If the phase shift, $\theta$, which was used to model the imperfect locking point of the EPR state reduced from $3 \pi / 180$ to $\pi / 120$, our model predicts that the range of the secure communication would extend further to $17 \mathrm{~km}$ for the RR protocol (Fig. S1(a)) and to more than $8 \mathrm{~km}$ for DR protocol (Fig. S1(b)). Achieving this level of quadrature squeezing and phase stability are experimentally challenging but feasible as up to $-12 \mathrm{~dB}$ of squeezing was reported previously [20].

\section{EXPERIMENTAL DETAILS AND MODELING OF P\&M WITH COHERENT STATES AND HOMODYNE DETEC- TION}

In this section, we discuss the experimental details, imperfections and modelling of the experiment with coherent states and homodyne measurements. A quantum noise limited $1064 \mathrm{~nm}$ laser was used in the experiment. A small portion of it was passed through a pair of phase and amplitude electro-optic modulators (EOMs). EOMs were used to provide a Gaussian distributed modulation on each quadrature. Each EOM was driven by an independent function generator, providing a broadband white noise signal up to $10 \mathrm{MHz}$. The magnitude of white noise was set to provide almost the same displacement on each quadrature. Outputs of function generators were divided into two. One part was sent to drive the EOMs and the other was recorded. This modulation record, after calibration, was Alice's data since she had control over the source. Here, calibration means determining the relationship between the function generator output and the phase space displacement as measured before transmission. We denote the variance of this phase space modulation $V_{S}$. The modulated beam was then sent through a lossy channel to Bob. To model the lossy channel, a vacuum state was introduced to the system and was mixed with the Bob's mode on a beamsplitter of transmission $T$. Upon receiving his mode, Bob performed a homodyne measurement, alternating between conjugate quadratures. An electronic delay was introduced to Alice's and Bob's data to gain the maximum correlation between them at 3.5-4.5 $\mathrm{MHz}$.

When the homodyne detector was locked to the phase quadrature there was $30 \mathrm{~dB}$ suppression of cross correlation between orthogonal quadratures and when it was locked to the amplitude quadrature the suppression was around $37 \mathrm{~dB}$. Our pair of detectors, both with dark noise clearance of $18 \mathrm{~dB}$, were balanced electronically, providing 30 $\mathrm{dB}$ of common mode rejection. Our homodyne efficiency was around $95 \%$ with fringe visibility of $98 \%$, limited by the mode distortions introduced by the EOMs. The photodiode's quantum efficiency was estimated to be around $98.5 \% .4 \times 10^{6}$ data points were sampled at $25 \times 10^{6}$ samples per second utilizing a digital data acquisition system. The process was repeated five times in order to provide sufficient statistics for each data points. These data were then digitally filtered to 3.5-4.5 MHz.

In order to find the maximum range over which the protocol provides secure communication, we wish to find the optimal modulation variance for each value of the channel transmission. We scanned the modulation variance over a range of 2 to 19 times the shot noise. As discussed in section B, by rescaling Alice's recorded signal, the key rates can be calculated using Eq. (S31) with reconciliation coefficient set to 0.95 .

To highlight the relative advantages of the coherent state source, consider the $\mathrm{CM}$ of the equivalent two mode EPR state:

$$
\operatorname{EPR}(s)=\left(\begin{array}{cccc}
\cosh (2 s) & 0 & \sinh (2 s) & 0 \\
0 & \cosh (2 s) & 0 & -\sinh (2 s) \\
\sinh (2 s) & 0 & \cosh (2 s) & 0 \\
0 & -\sinh (2 s) & 0 & \cosh (2 s)
\end{array}\right)
$$

where $s$ is the squeezing parameter which is related to the modulation variance via $\cosh (2 s)=V_{S}+1$. To model the prepare $\&$ measure experiment, we remain in the equivalent EB picture and begin with $\gamma_{\text {in }}=\operatorname{EPR}(\mathrm{s})$. Recall that one part of the equivalent EPR state was sent to Bob through a lossy channel where he performed a homodyne detection, and on the other part Alice performed a heterodyne detection. Although much more flexible, the coherent state setup naturally still suffers from imperfections which in turn effect the optimum modulation. These imperfect correlations arise partly from cross correlation between orthogonal quadratures and partly from our limited ability to maximize the correlation between Alice and Bob's modes using electronic delay. Both phenomena can be thought of as an unknown rotation in the system. A rotation operator with small angles is applied to the $X$ and $P$ quadratures of the second mode (Bob's mode) to model the imperfect correlation between Alice's and Bob's modes.

The channel transmission, $T$, can again be determined directly by taking the ratio of the correlation at a particular setting with the correlation at full transmission. Technically, the experimental channel would also introduce a small amount of excess noise, however this is negligible compared to the excess noise coming from the effects described above. The final simulated covariance matrix hence is

$$
\gamma_{\text {out }}=S\left[\gamma_{\text {in }} \oplus V_{\chi}(B) \oplus \operatorname{diag}(1,1)\right] S^{T},
$$

where $S$ is given by

$$
S=R T\left(\theta_{x}, \theta_{p}\right) B S_{1,4}(1 / 2) B S_{2,3}(T) .
$$

Here, $V_{\chi}=\operatorname{diag}\left(1+\chi_{x}, 1+\chi_{p}\right)$, where $\chi_{x(p)}$ is the excess noise in $\hat{x}(\hat{p})$ quadrature. The rotation matrix,

$$
R T\left(\theta_{x}, \theta_{p}\right)=\left(\begin{array}{cc}
\cos \theta_{x} & \sin \theta_{x} \\
-\sin \theta_{p} & \cos \theta_{p}
\end{array}\right)
$$

serves as the fitting parameter to model the unknown rotation due to aforementioned experimental imperfection.

To evaluate the key rate Eq. (S31), we neglect the excess noise in the channel by setting $\chi_{x(p)}=0$. The variation of key rates versus the equivalent modulation squeezing parameter for 5 different transmissions is shown in Fig. S2(a). As the modulation is increased, so too is the detrimental effect on the correlations, leading to a smaller value for the optimal modulation parameter, whereas for an ideal experiment, this would depend only upon $\beta$. In inset(b) of Fig. S2 the gap between the ideal case without cross correlation and the realistic case is shown for the case of perfect transmission. For each transmission value, the modulation squeezing parameters that provide the highest key rate are chosen and plotted in Fig. S2(c). These optimum variances match well with what our theoretical model predicts. As is clear from Fig. S2(a), using coherent states provides a much greater range over which to tune the equivalent squeezing. When using actual EPR states the maximum 
achievable value for $s$ is around 0.8 , well short of the optimum. Our model also predicts that if the cross correlation between Alice and Bob's modes was zero, the range of secure communication for this protocol would extend from $4.5 \mathrm{~km}$ to $6.5 \mathrm{~km}$ as depicted in Fig. S2(c). topology for gravitational wave detection," Phys. Rev. Lett. 104, 251102 (2010).

\section{REFERENCES}

1. I. Białynicki-Birula and J. Mycielski, "Uncertainty relations for information entropy in wave mechanics," Commun.Math. Phys. 44, 129-132 (1975).

2. H. Maassen and J. Uffink, "Generalized entropic uncertainty relations," Phys. Rev. Lett. 60, 1103-1106 (1988).

3. M. Berta, M. Christandl, R. Colbeck, J. Renes, and R. Renner, "The uncertainty principle in the presence of quantum memory," Nature Phys. 6, 659-662 (2010).

4. M. Berta, F. Furrer, and V. B. Scholz, "The smooth entropy formalism on von Neumann algebras," arXiv:1107.5460 (2011).

5. F. Furrer, M. Berta, M. Tomamichel, V. B. Scholz, and M. Christandl, "Position-momentum uncertainty relations in the presence of quantum memory," Journal of Mathematical Physics 55, 122205 (2014).

6. A. Ferenczi, "Security proof methods for quantum key distribution protocols," PhD Thesis, University of Waterloo (2013).

7. R. L. Frank and E. H. Lieb, "Extended Quantum Conditional Entropy and Quantum Uncertainty Inequalities," Communications In Mathematical Physics 323, 487-495 (2014).

8. T. C. Ralph, "Continuous variable quantum cryptography," Phys. Rev. A 61, 010303 (1999).

9. M. Hillery, "Quantum cryptography with squeezed states," Phys. Rev. A 61, 022309 (2000).

10. F. Grosshans, G. V. Assche, J. Wenger, R. Brouri, N. J. Cerf, and P. Grangier, "Quantum key distribution using Gaussian-modulated coherent states," Nature 421, 238-41 (2003).

11. C. Weedbrook, A. Lance, W. P. Bowen, T. Symul, T. C. Ralph, and P. K. Lam, "Quantum cryptography without switching," Phys. Rev. Lett. 93, 170504 (2004).

12. C. Weedbrook, A. Lance, W. Bowen, T. Symul, T. C. Ralph, and P. K. Lam, "Coherent-state quantum key distribution without random basis switching," Phys. Rev. A 73, 022316 (2006).

13. F. Grosshans and P. Grangier, "Continuous variable quantum cryptography using coherent states," Phys. Rev. Lett. 88, 057902 (2002).

14. M. Reid, "Quantum cryptography with a predetermined key, using continuous-variable Einstein-Podolsky-Rosen correlations," Phys. Rev. A 62, 062308 (2000).

15. F. Grosshans, N. J. Cerf, P. Grangier, J. Wenger, and R. TualleBrouri, "Virtual entanglement and reconciliation protocols for quantum cryptography with continuous variables," Quantum Inf. Comput. 3, 535-552 (2003).

16. R. García-Patrón and N. J. Cerf, "Unconditional optimality of Gaussian attacks against continuous-variable quantum key distribution," Phys. Rev. Lett. 97, 190503 (2006).

17. M. Navascués, F. Grosshans, and A. Acín, "Optimality of Gaussian attacks in continuous-variable quantum cryptography," Phys. Rev. Lett. 97, 190502 (2006).

18. I. Devetak and A. Winter, "Distillation of secret key and entanglement from quantum states," Proc. R. Soc. A 461, 207 (2005).

19. C. Weedbrook, S. Pirandola, R. García-Patrón, N. J. Cerf, T. C. Ralph, J. Shapiro, and S. Lloyd, "Gaussian quantum information," Rev. Mod. Phys. 84, 621-669 (2012).

20. T. Eberle, S. Steinlechner, J. Bauchrowitz, V. Händchen, H. Vahlbruch, M. Mehmet, H. Müller-Ebhardt, and R. Schnabel, "Quantum enhancement of the zero-area Sagnac interferometer 

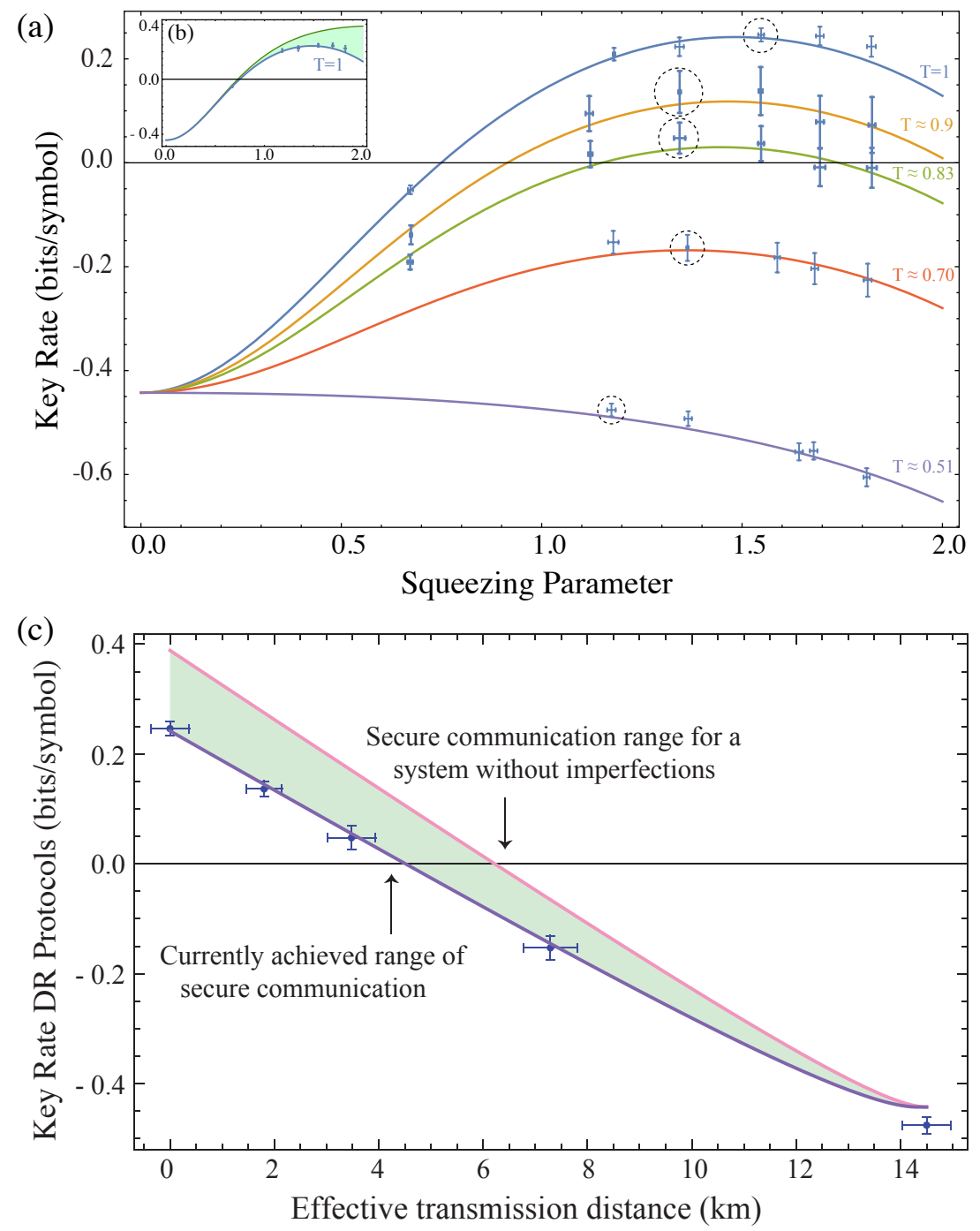

Fig. S2. (a) Variation of key rates versus effective modulation squeezing parameter for 5 different transmissions. A theory line with the average transmission of the channel is fitted on the experimental data points with 1 s.d. error bars. Data points surrounded by dashed circles correspond to the optimum modulation squeezing parameters which result in the highest key rate for each transmission. The key rates resulting from these optimum modulation variances are shown separately in (c). Inset (b) demonstrates the gap between the theoretical cross-talk free model and the realistic model which captures the experimental imperfections. (c) Predicted improvement of secure transmission distance through the optical fibre for the coherent state protocol with an improved experimental setup (red curve). The model for the current system (blue curve) is plotted along with experimental data (blue points) for comparison. In the actual experiment, the optimal modulation variance is reduced due to unwanted cross-quadrature correlations. In the improved setup, the cross-talk has been eliminated and the optimal modulation variance is now determined by the reconciliation efficiency, which is chosen to be 0.95 for both cases. These theoretical lines are produced using the model described in section 5 , where the value of the unknown rotation, $\left(\theta_{x}, \theta_{p}\right)$, was estimated to be about $\approx(6 \pi / 180,3 \pi / 180)$ and equation (S31). 